\title{
ArcheoSciences
}

Revue d'archéométrie

36 | 2012

Varia

\section{L'agglomération antique de Fontaine-les-Bassets (Orne, France) : apports des recherches récentes} sur un site oublié

The Roman Small Town of Fontaine-les-Bassets (Orne, France): A New Look at a

Forgotten Site; Evidence from Recent Research

\section{Sophie Quevillon}

\section{OpenEdition \\ Journals}

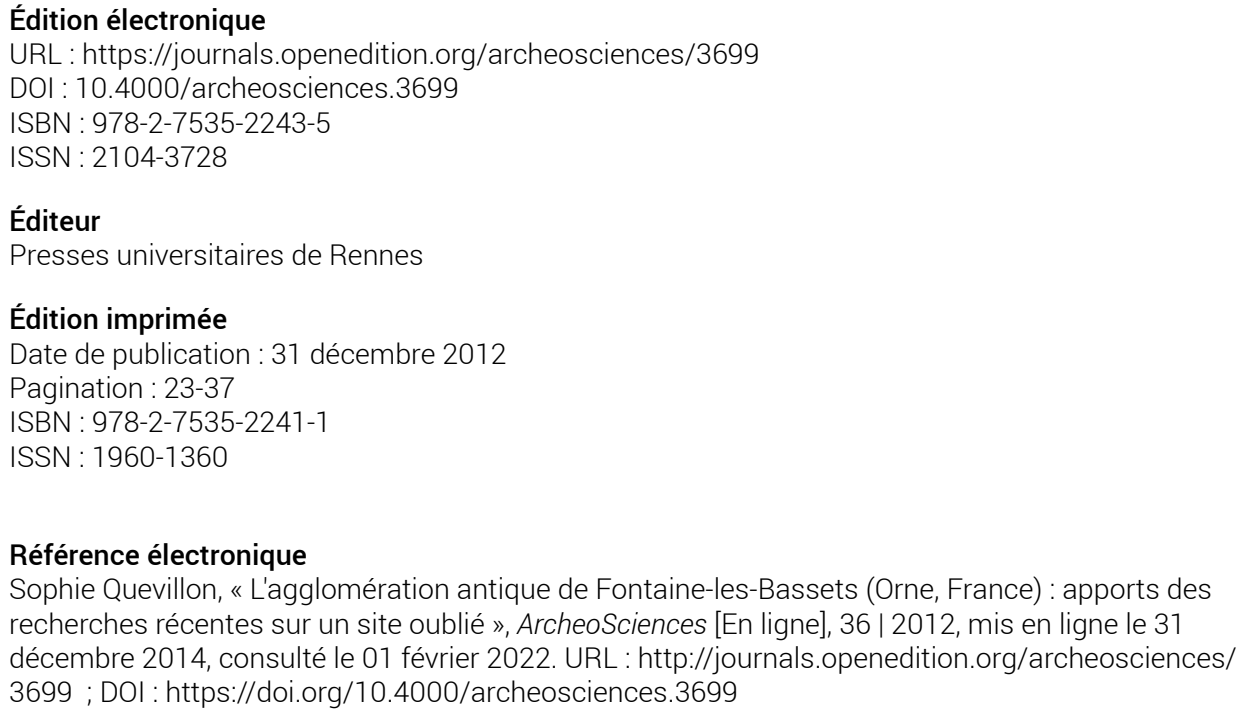




\title{
L'agglomération antique de Fontaine-les-Bassets (Orne, France) : apports des recherches récentes sur un site oublié
}

\author{
The Roman Small Town of Fontaine-les-Bassets (Orne, France): \\ A New Look at a Forgotten Site; Evidence from Recent Research
}

\author{
Sophie Quevillon*
}

\begin{abstract}
Résumé : Situé dans l'actuel département de l'Orne, le site de Fontaine-les-Bassets est une agglomération antique étendue sur près de 30 hectares. Identifiée en 1989 par la prospection aérienne mais jamais fouillée, elle est à nouveau étudiée dans le cadre d'un programme de recherche visant à mieux connaître sa formation et son organisation durant l'antiquité. En 2009 et 2010, une prospection géophysique (méthode électrique), associée à une prospection pédestre ainsi qu’à la mise en place d'un Système d'Information Géographique, a été réalisée sur 20 hectares livrant une image particulièrement riche et complexe de l'agglomération. En effet, un ensemble d'édifices, vraisemblablement monumentaux, constitue le cœur de cette agglomération autour duquel se déploient des îlots bâtis à l'intérieur d'un réseau de rues quadrillées.
\end{abstract}

Abstract: Located in the present Orne department, the site of Fontaine-les-Bassets is a Roman town covering nearly 30 hectares. Identified in 1989 by aerial photography but never excavated, we are now taking a fresh look at the town as part of a research program aiming at understanding its structure and organization during the Roman period. In 2009, a new spatial approach was started including geophysical survey (Automatic Resistivity Profiling), field survey and Geographic Information System analysis. The geophysical investigation, covering 20 hectares, revealed a rich and complex urban centre. Indeed, a monumental group of buildings very probably forms the heart of the town, around which develop blocks within an orthogonal street network.

Mots clé : agglomération antique, Basse-Normandie, prospection électrique, prospection aérienne, prospection pédestre, système d'information géographique (SIG).

Keywords: Gallo Roman town, Lower Normandy, resistivity survey, aerial photography, field survey, geographical information systems (GIS).

\section{Présentation du Site ET HISTORIQUE DES RECHERCHES}

Aujourd'hui petite commune rurale de l'Orne, à 15 kilomètres au sud-est de Falaise, Fontaine-les-Bassets était, à l'époque antique, une agglomération étendue sur près de 30 hectares sur le passage d'une voie reliant Bayeux à
Chartres. Située en périphérie de la zone actuellement habitée, sous des terres agricoles depuis longtemps cultivées, l'agglomération semble ne pas avoir perduré au-delà de la période antique.

Mentionné pour la première fois en 1788 , le site n'a jamais fait l'objet de fouilles archéologiques et ce malgré les

* Ministère de la Culture, Service régional de l'archéologie de Basse-Normandie - 13 bis rue St-Ouen, 14052 CAEN cedex 4; CNRS, UMR 6566, CReAAH; Université de Rennes 1 - 263, Avenue du général-Leclerc, Campus de Beaulieu CS 74205, 35042 Rennes cedex. (sophie.quevillon@culture. gouv.fr) 
nombreux vestiges qui semblent être restés visibles dans le paysage jusqu’à la fin du XIX si ècle.

En 1989, la physionomie de l'agglomération antique est enfin dévoilée grâce à la photographie aérienne. En 1996, une première prospection pédestre permet de confirmer l'existence d'une occupation antique et par la même occasion de disposer d'un échantillon de mobilier sur ce site méconnu (Leclerc, 1996).

La volonté d'entreprendre de nouvelles recherches sur l'agglomération antique de Fontaine-les-Bassets s'inscrit dans une réflexion régionale sur l'émergence du fait urbain à travers le développement et la structuration d'un réseau d'agglomérations et le maillage d'un territoire entre agglomérations dites « secondaires » et chefs-lieux de cité (fig. 1).
L’objectif principal de l'opération, initiée en 2009, était avant tout d'obtenir de nouvelles données précisant l'étendue mais aussi l'organisation interne (réseau viaire, bâtiments...) de cette agglomération. Le souhait de recourir à des méthodes d'étude non destructives a orienté le choix vers une stratégie articulée autour des méthodes de prospection (aérienne, pédestre et géophysique). La confrontation de ces trois méthodes permettra une analyse spatiale du site mais aussi l'implantation raisonnée et ciblée de sondages archéologiques.

Afin de disposer d'une vision d'ensemble la plus complète possible, une prospection utilisant la méthode de la résistivité électrique a été mise en place en 2009 et 2010. En parallèle, une prospection pédestre a été menée sur l'em-

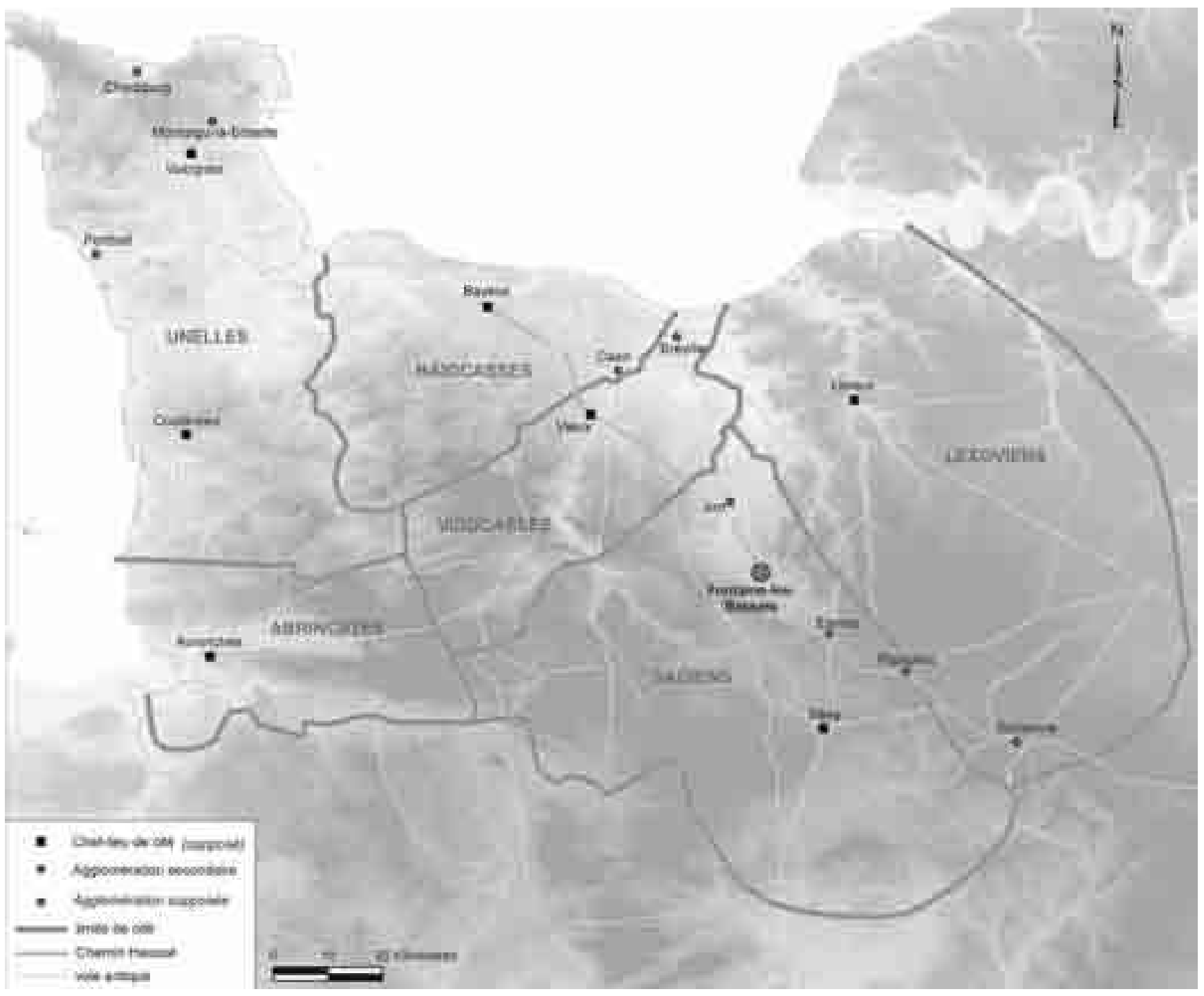

Figure 1 : Cités, chefs-lieux et agglomérations antiques en Basse-Normandie.

Figure 1: Cities, administrative centres and Roman small towns in Lower Normandy. 
prise présumée de l'agglomération ainsi que dans un rayon d'environ un kilomètre autour du centre urbain de manière à identifier une éventuelle continuité de l'occupation au-delà des 20 hectares déjà connus. Les éléments céramiques récoltés en 1996, puis en 2009-2010, indiquent une occupation depuis le milieu du $\mathrm{I}^{\text {er }}$ siècle apr. J.-C. jusqu'au courant du $\mathrm{III}^{\mathrm{e}}$ siècle avec toutefois quelques rares éléments plus tardifs (IV ${ }^{\mathrm{e}}-\mathrm{V}^{\mathrm{e}}$ siècles). Un relevé systématique du mobilier a été effectué à l'aide d'un GPS différentiel afin d'observer d'éventuelles concentrations. L'ensemble des informations ainsi recueillies a été intégré et analysé à l'aide d'un Système d'Information Géographique (SIG).

À l'instar de nombreuses autres expériences récentes réalisées sur des sites semblables (Le Vieil-Evreux : Dabas et al., 2005; Mandeure-Mathay : Thivet et al., 2009; Les ToursMirandes : Dieudonné-Glad, 2010), la méthode électrique a révélé une image particulièrement riche de l'agglomération antique de Fontaine-les-Bassets, confirmant les hypothèses émises par le passé à partir des photographies aériennes.

\section{Problématique et méthodologie}

À Fontaine-les-Bassets, l'agriculture intensive ainsi que les nombreuses prospections clandestines constituent une menace sérieuse pour le potentiel scientifique du site et l'étude de cette ville antique disparue. Le programme de recherche mis en place aujourd'hui affiche donc un double objectif, à la fois de connaissance historique et de préservation des vestiges archéologiques. Si ces derniers semblent préservés en grande partie dans une parcelle d'environ 23 hectares, actuellement en culture, l'occupation antique s'étend toutefois au-delà de cet espace, faisant de Fontaineles-Bassets une agglomération rivalisant, en superficie, avec les chefs-lieux de cités connus pour l'antiquité en BasseNormandie (Bayeux, Lisieux ou Vieux).

Si l'emprise couverte par les trois méthodes de prospection n'est pas identique, elle concerne cependant pour chacune le secteur le plus essentiel, à savoir les 23 hectares de la grande parcelle (YA 265) renfermant le cœur de l'agglomération (fig. 3).

\section{La prospection aérienne}

La Plaine de Caen-Argentan fait depuis de nombreuses années l'objet de prospections aériennes régulières, principalement menées par J. Desloges (Desloges, 1994, 2001, 2011, Desloges et al., 1993). Ces survols intensifs ont permis de disposer d'un riche corpus photographique, notamment sur l'agglomération antique de Fontaine-les-Bassets. Celui-ci documente à la fois l'espace urbain car les premiers clichés ont permis l'identification d'un complexe monumental au centre de la parcelle, mais aussi l'environnement immédiat de la ville antique et notamment les occupations protohistoriques.

Le récolement de l'ensemble des clichés disponibles depuis 1989 a permis de constituer un corpus varié sur le site, dont une partie a pu être intégrée au SIG (fig. 2). Depuis 2009 et la reprise des recherches, une attention particulière est portée aux indices phytographiques lors de survols ciblés.

Même si tous les clichés aériens disponibles n'ont pu faire l'objet d'un géoréférencement, les vues les plus générales apportent de nombreuses informations sur la ville et principalement sur l'organisation et l'extension de son réseau viaire (notamment pour les parcelles YB 249 et ZB 563, fig. 2). Un récent cliché, pris en 2011 , a permis d'observer et de géoréférencer pour la première fois de nouveaux éléments de rues à l'est de l'agglomération, secteur encore non couvert par la prospection électrique (fig. 7).

C'est après le géoréférencement des photographies aériennes en 2009 qu'une première zone d'évaluation a été déterminée pour réaliser une prospection électrique. Le secteur monumental central, le mieux documenté par la prospection aérienne, a été choisi pour confirmer ou infirmer la pertinence de la méthode électrique sur ce site. La qualité des résultats obtenus en 2009 après prospection de 5,7 hectares, illustrant une forte densité d'anomalies de résistivité, a encouragé la poursuite des recherches en 2010 par le biais de cette méthode.

\section{La prospection pédestre}

À Fontaine-les-Bassets, la collecte d'objets antiques est une tradition locale car depuis des décennies les habitants du village et des alentours n'ont eu de cesse de ramasser l'abondant mobilier archéologique mis au jour par les labours.

La prospection pédestre mise en œuvre dans le cadre de ce projet de recherche s'est quant à elle voulue méthodique. Réalisée en 2009 et en 2010, elle a dans un premier temps cherché à étudier la répartition des artefacts à l'emplacement présumé de la ville antique (parcelle YA 265) pour, dans un second temps, tenter d'évaluer l'étendue de l'occupation dans les parcelles environnantes.

Les prospections pédestres conduites dans les parcelles contiguës à l'agglomération ont été plutôt pauvres en information car aucune concentration de mobilier significative n'a été observée, confirmant ainsi l'hypothèse d'une ville inscrite à l'intérieur d'un quadrilatère formé par les parcelles YA 265, 246 et 247 (fig. 3). Malgré les indices de voirie bien visibles sur les photographies aériennes dans 


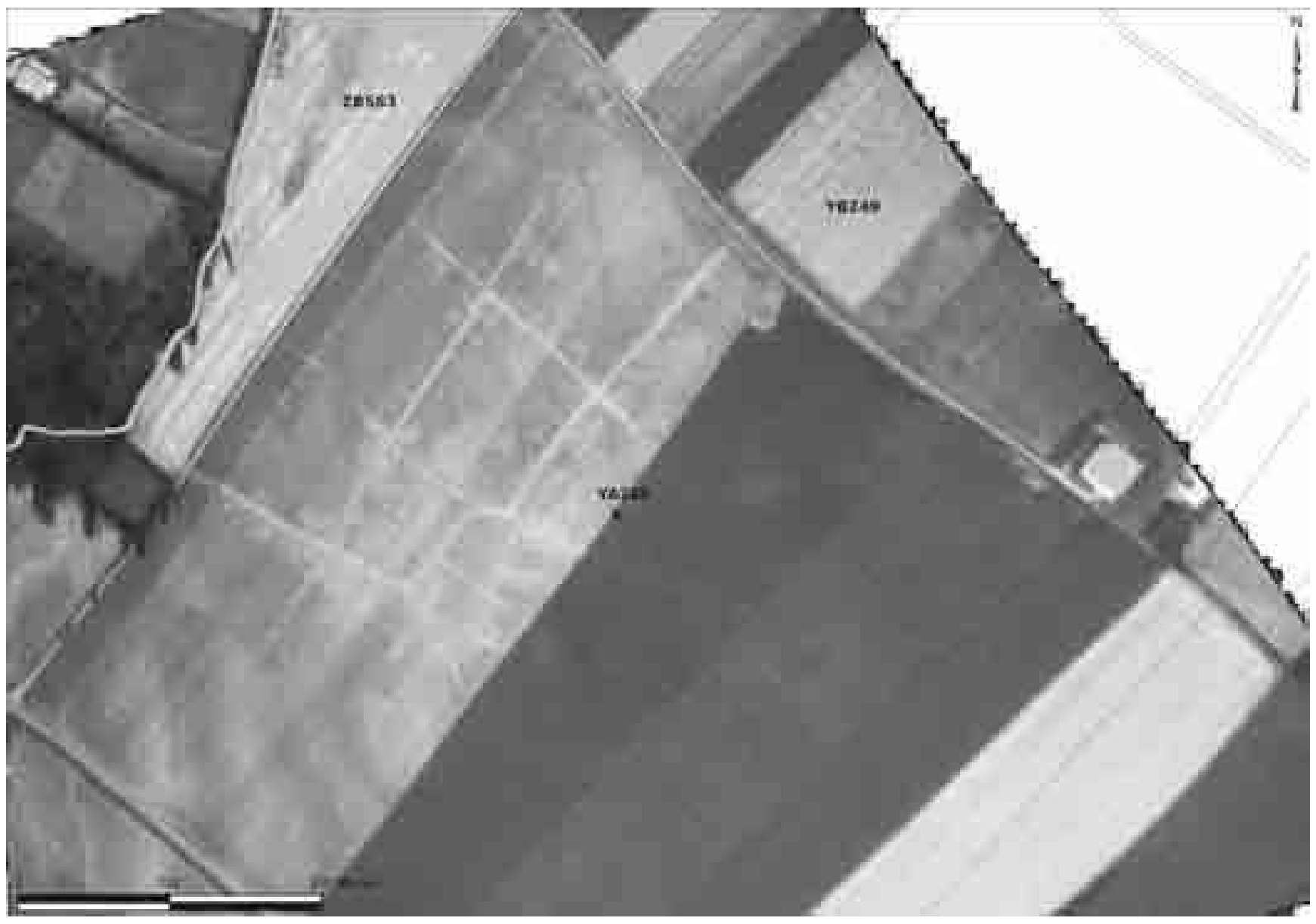

Figure 2 : L'agglomération antique de Fontaine-les-Bassets, vue du sud-ouest, lors de sa redécouverte par la prospection aérienne en 1989 (cliché : G. Guillemot).

Figure 2: The Roman small town of Fontaine-les-Bassets, seen by the southwest, during its rediscovery by aerial photography in 1989.

deux parcelles voisines au nord (YB 249) et à l'ouest (ZB 563) (fig. 2), aucun mobilier significatif n’y a été découvert. Quelques fragments épars de terres cuites architecturales (briques, tuiles) restent perceptibles dans un rayon d'environ 500 mètres au nord et à l'est du site mais aucun autre élément caractéristique ne permet d'attester une réelle occupation pérenne (ces fragments résiduels pouvant être issus d'épandages récents). Enfin, la présence d'herbages, de parcelles non cultivées ou bien d'activités modernes d'extraction de calcaire (par exemple au sud dans la parcelle YA 274) a rendu impossible la prospection de certaines parcelles autour de l'agglomération antique.

Ainsi, en 2009, un cheminement régulier de lignes espacées tous les 20-25 mètres a couvert la superficie supposée de l'agglomération (environ 6 parcelles, soit 42 hectares). En 2010, la prospection s'est étendue aux parcelles conti- guës à l'agglomération antique, soit 38 parcelles parcourues (115 hectares cumulés pour 2009 et 2010) (fig. 3).

En parallèle à son échantillonnage, le mobilier de surface a été enregistré à l'aide d'un GPS différentiel. L'intérêt de l'utilisation de cet outil en prospection archéologique n'est aujourd'hui plus à démontrer tant il facilite et simplifie la localisation et l'enregistrement des éléments découverts (Rodier et Saligny, 2006; Campana, 2006). De plus, son intégration dans la chaîne opératoire archéologique, depuis l'acquisition de l'information sur le terrain jusqu'à son analyse au sein du SIG, en fait un outil indispensable.

Les campagnes de prospection pédestre ont été précédées par l'élaboration d'une base de données spécifique intégrée au carnet de terrain du GPS et associée au fond cadastral actuel de la commune. L'enregistrement typologique du mobilier a ainsi été facilité, de même que le traitement des 
Figure 3 : Prospection pédestre 2009 et 2010 : parcelles prospectées et positionnement GPS du mobilier archéologique prélevé.

Figure 3: Field survey 2009 and 2010: parcels surveyed and GPS recording of the archaeological artifacts collected.

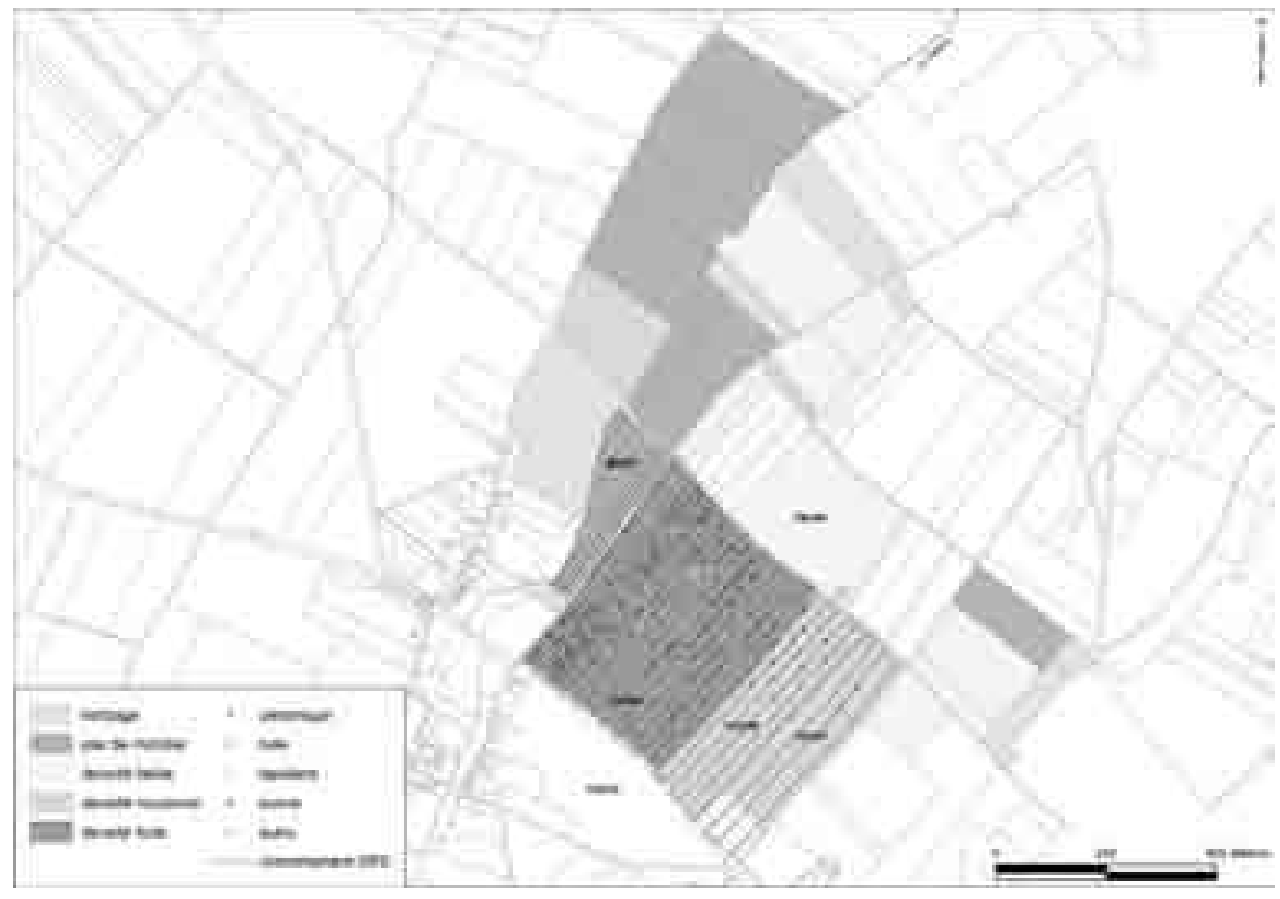

données au retour du terrain. Le matériel à disposition, un GPS Trimble Pro XRS, a été utilisé en mode différentiel grâce à l'implantation d'une station de base servant de référence au récepteur mobile en charge de l'enregistrement des données. Cette configuration a permis d'obtenir une précision horizontale de la localisation comprise entre 20 et $50 \mathrm{~cm}$ et une précision verticale entre 25 et $80 \mathrm{~cm}$ (à $99 \%$ de précision pour l'ensemble des données).

\section{La prospection électrique}

Le choix de la méthode géophysique, en complément des prospections aériennes et pédestres, s'est imposé dès lors que celle-ci permettait d'explorer une vaste surface, à la fois sans destruction mais également en un court laps de temps. À Fontaine-les-Bassets, la méthode électrique ARP(C) ${ }^{1}$ a été choisie en raison d'un bon contraste des propriétés électriques des matériaux présents (substrat calcaire, structures maçonnées, encaissant limoneux) et de la possibilité de prospecter rapidement de grandes surfaces avec une résolution suffisante (plus de $4 \mathrm{ha} /$ jour). Bien adaptée à la mise en évidence de constructions en pierres ou en briques cuites, plus résistantes dans un encaissant fin, la méthode mesure la résistivité électrique apparente du sol, soit la difficulté

1. Prospection électrique, méthode ARP@ (Automatic Resistivity Profiling) : acquisition et traitement des données réalisés par la société GEOCARTA (A. Badoc, T. Jubeau, R. Makhlouf). du courant électrique à traverser un matériau. Le matériel utilisé dans le cadre de cette étude est le dispositif multipôle ARPC (Geocarta) (Dabas, 2006) permettant de réaliser des mesures jusqu'à 50,100 et $170 \mathrm{~cm}$ de profondeur selon un pas de mesure de $10 \mathrm{~cm}$ et des profils tous les mètres. La rapidité d'acquisition des données, grâce à l'utilisation d'un quad qui permet de réaliser des profils espacés d'un mètre perpendiculairement au sens des cultures, et la précision du système GPS embarqué (GPS différentiel bi-fréquentiel ou GPS RTK) rendent la méthode extrêmement rapide et efficace. Ainsi, ce sont près de 20 hectares qui ont pu être couverts durant les campagnes menées en 2009 (5,7 ha) et 2010 (14 ha) (fig. 4). Trois cartes présentant les résultats ont été établies selon les trois profondeurs mesurées $(0-50 \mathrm{~cm}$, 0-100 cm et 0-170 cm). L'ensemble des données montre un nombre très important d'anomalies essentiellement résistantes, en particulier au centre de la parcelle. La carte à $0-50 \mathrm{~cm}$ est celle qui présente le plus d'éléments de détail malgré la lecture plus difficile de certains secteurs. Ces résultats hétérogènes semblent principalement liés à de fortes perturbations du terrain, à la fois géologiques et anthropiques (labourages profonds et intensifs), de même qu'à un probable mauvais état de conservation des structures. Des effets liés aux pratiques agricoles modernes (compaction du sol, sillons, passage des tracteurs...) sont visibles sur l'ensemble des cartes, mais des filtres directionnels ont été appliqués lors du traitement des données pour limiter ces biais. Les varia- 


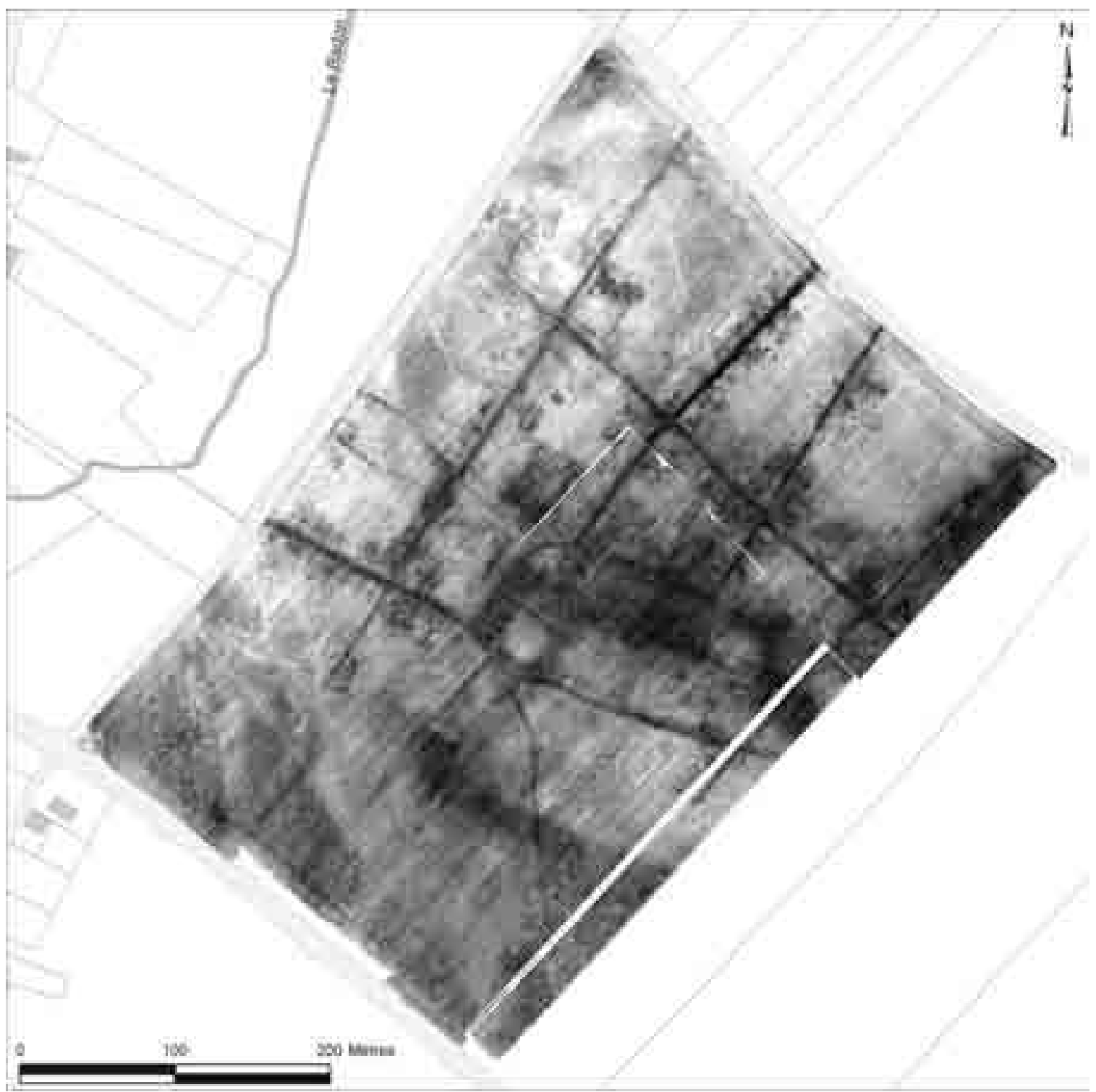

Figure 4 : Image des résultats des prospections électriques menées en 2009 et 2010 couvrant 20 hectares de la parcelle principale (YA 265).

Figure 4: Image of the results of the resistivity survey conducted in 2009 and 2010 covering 20 hectares of the main parcel (YA 265).

tions à grande longueur d'onde de la résistivité apparente sont à associer à des changements pédologiques. Toutefois, les données acquises sur cette parcelle sont, de manière générale, de bonne qualité et n'ont fait l'objet que d'un léger filtrage (retrait des valeurs nulles sur les fichiers bruts résultant d'une perte de contact des électrodes avec le sol et médiane en 2 dimensions sur une fenêtre de 1,5 x 1,5 m avec un seuil de $10 \%$ sur la grille).

\section{La mise en place du Système d'Information Géographique}

Un SIG, outil indispensable à l'analyse de la topographie urbaine, a été mis en place dès la reprise des recherches sur le site. Il rassemble toutes les données acquises depuis les premières photographies aériennes jusqu'aux images issues de la prospection électrique. Conçu à l'aide du logiciel ArcGis 9.3 (ESRI ()), il a été élaboré dans l'objectif d'intégrer l'ensemble des données disponibles ainsi que celles 
nouvellement acquises afin d'en faciliter l'analyse et la compréhension. Ainsi, les premiers travaux de cartographie ont essentiellement consisté en une confrontation des différentes sources de données. Une partie des clichés aériens a pu être géoréférencée et intégrée à l'espace étudié. Un grand nombre d'entre eux, de par le mauvais état de conservation du support ou leur absence de point de référence utilisable, n'a pu être positionné mais garde toutefois une précieuse valeur informative. Les relevés GPS, intégrés après correction différentielle, offrent une information sur la répartition du mobilier archéologique en surface.

Enfin, les données de la prospection électrique viennent compléter le corpus des informations intégrées au SIG. Une dernière campagne, prévue en 2012, achèvera la couverture du site par la méthode électrique. Une première cartographie, encore à l'état d'ébauche, a été tentée afin d'identifier les types de structures archéologiques à partir du croisement de l'ensemble des données.

\section{RÉsultatS DES PROSPECTIONS ÉLECTRIQUES}

L'interpolation de l'ensemble des données de la prospection électrique (maille de $50 \mathrm{~cm} \times 50 \mathrm{~cm}$ ) fait apparaître une forte densité d'anomalies résistantes au centre de la parcelle prospectée par opposition au secteur sud qui présente des anomalies conductrices organisées de manière radiale (Fig. 4). L'association de celles-ci à des structures archéologiques reste difficile et laisse penser qu'il s'agirait d'un autre type de vestiges ou de vestiges n'appartenant pas à la période antique. La comparaison avec les données de prospection pédestre qui, dans ce secteur, ont livré une grande quantité de scories de fer, suggère pour cette zone un type d'occupation de nature différente de celle du reste de la parcelle. Une prospection magnétique livrerait peut-être d'autres informations. D'autres anomalies linéaires conductrices, localisées principalement dans le quart nord-ouest de la parcelle, pourraient correspondre à des cheminements, soit antérieurs à l'antiquité, soit constitués de matériaux de nature différente (fig. 4).

\section{Réseau viaire}

D’un point de vue général, les anomalies les plus remarquables et les plus facilement identifiables sont les fortes anomalies résistantes linéaires qui traversent la parcelle. Elles peuvent être identifiées comme appartenant au réseau viaire de l'agglomération et viennent compléter, avec plus de précision, les éléments observés sur les clichés aériens. Quatre axes principaux orientés nord-est/sud-ouest ainsi que deux axes nord-ouest/sud-est divisent l'espace urbain en îlots irréguliers (fig. 5). Le tracé linéaire le plus fortement résistant correspond à l'axe nord-est/sud-ouest (C) attesté par prospection aérienne dans sa prolongation au nord (parcelle YB 249). D'une largeur maximale estimée à 5 mètres, il semble révéler une chaussée particulièrement bien conservée et vraisemblablement empierrée (l'hypothèse d'une voie dallée est peu envisageable compte tenu du contexte régional qui ne compte qu'un seul exemple de ce type dans le cheflieu de cité des Lexoviens, à Lisieux). Les cinq autres axes principaux semblent présenter des largeurs plus variées et apparaissent moins résistants que l'axe $\mathrm{C}$.

À l'intérieur de ce réseau principal, on observe un grand nombre de tracés linéaires résistants d'orientation et de largeur variable (entre 2 et 5 mètres) menant pour certains vers des anomalies identifiées comme des ensembles bâtis, pour d'autres sortant de l'espace aggloméré. Certains clichés aériens peuvent aujourd'hui compléter ce réseau, notamment en ce qui concerne les axes $\mathrm{E}$ et $\mathrm{F}$ tant dans la direction de l'ouest que de l'est. L'identification des anomalies linéaires résistantes associées à un réseau secondaire de circulation est difficile à établir pour le centre de l'agglomération qui apparaît fortement résistant et pour lequel la largeur des tracés ne peut être connue avec précision. L’orientation des axes secondaires semble cependant suivre celle des deux grands axes principaux nord-ouest/sud-est ( $\mathrm{E}$ et $\mathrm{F}$ ) mais leur moindre résistivité électrique laisse supposer des matériaux ou un traitement différent de la chaussée.

Un calcul simple sur l'orientation de ces axes (par rapport au nord géographique), réalisé à l'aide du SIG, a mis en valeur la prédominance de certaines orientations préférentielles :

$-32^{\circ}$ : axes $\mathrm{A}$ et $\mathrm{B}$

$-43^{\circ}$ : axes $\mathrm{C}$ et $\mathrm{D}$

$-133^{\circ}$ : axes E, G, H, Chemin Haussé.

Peu de carrefours apparaissent strictement orthogonaux, car bien que les axes principaux $\mathrm{A}, \mathrm{B}, \mathrm{C}$ et $\mathrm{D}$ présentent un tracé rectiligne, les axes $\mathrm{E}, \mathrm{F}$ et $\mathrm{G}$ sont convergents et tendent à se rejoindre à l'est de l'agglomération en un point encore non connu par les données de prospection électrique. Seules les intersections de l'axe $\mathrm{C}$ avec E, H, L ainsi qu'avec le Chemin Haussé, de même que celle entre les axes $\mathrm{D}$ et $\mathrm{E}$ sont relativement orthogonales.

L'articulation des voies de circulation avec les constructions reste à explorer afin de mieux comprendre l'organisation de la circulation. De même, l'importance des espaces ouverts, empierrés ou non (places?), doit être prise en considération dans l'étude des espaces publics associés aux différentes constructions. 


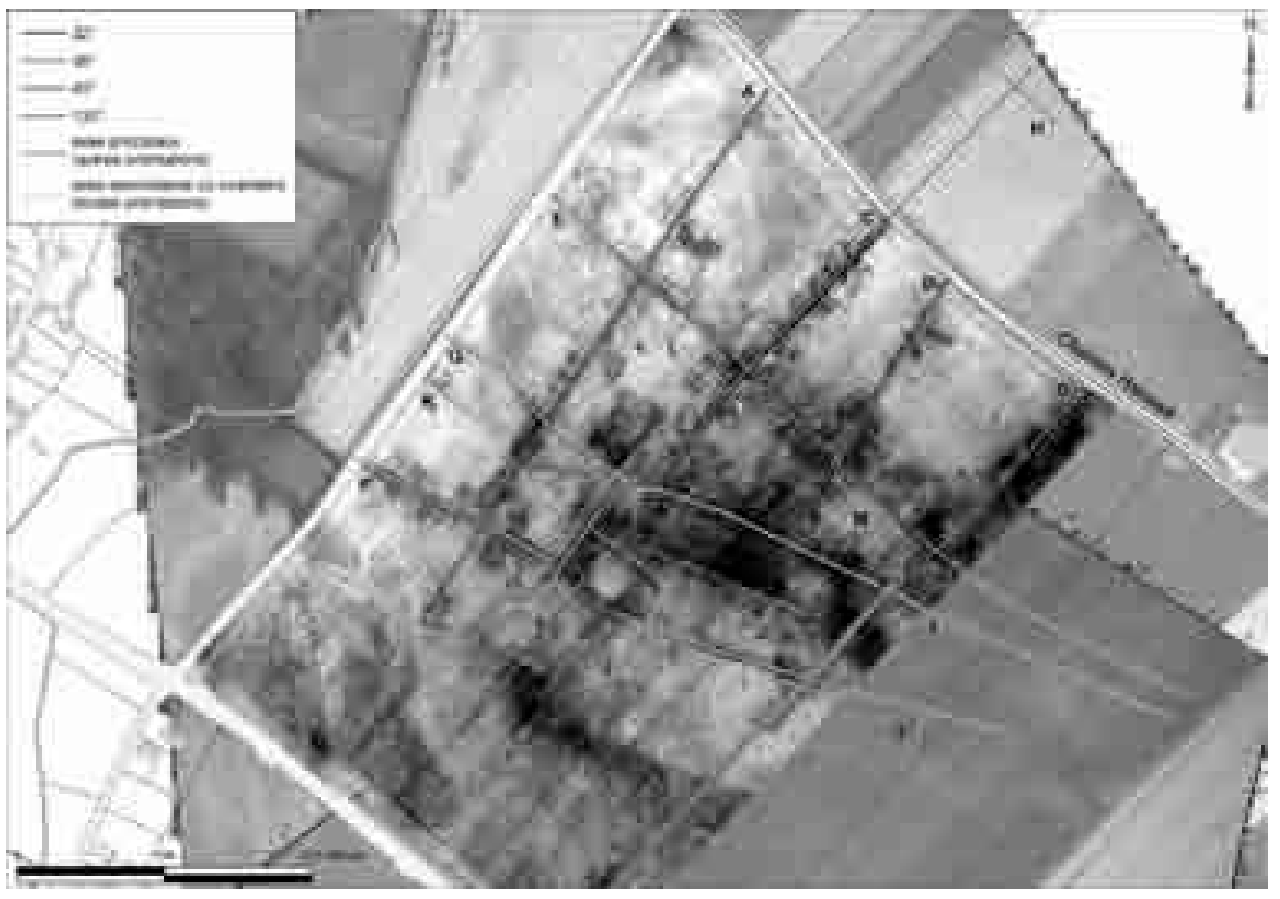

Figure 5 : (Voir planche couleur) Restitution de la trame viaire à partir des prospections électrique et aérienne et calcul des orientations.

Figure 5: (See colour plate) Restitution of the street network using resistivity survey and aerial photography along with calculation of city axes and street orientations.

\section{Constructions}

Deux catégories d'anomalies résistantes, définies par leur forme et leur taille, peuvent être associées à deux types de constructions (fig. 6) :

- les grands bâtiments et ensembles de bâtiments

- les petits bâtiments diffus ou isolés.

Le centre de la parcelle est la zone la plus dense en anomalies résistantes et ce pour les trois profondeurs d'investigation. Toutefois, la forte résistivité de ce secteur rend l'identification des structures archéologiques très difficile. Ce phénomène pourrait, a priori, être expliqué par la présence importante de matériaux de construction, notamment de pierres, matérialisée soit par des constructions maçonnées encore en élévation, soit par une accumulation d'épais niveaux de destruction.

Dans la catégorie des grands bâtiments ou grands ensembles de bâtiments, trois anomalies fortement résistantes se démarquent, y compris sur la carte de profondeur 0-170 cm. Il s'agit de grands ensembles complexes dont l'un, le plus au sud (bât. 1), avait déjà été identifié lors des campagnes de prospection aérienne. De forme quadrangulaire, il présente une longueur d'environ 45 mètres pour une largeur d'environ 35 mètres. Quatre axes de circulation convergent vers cette imposante construction et semblent s'arrêter à son contact (présence d'une place sur la façade sud?). À une cinquantaine de mètres au nord-ouest, un deuxième ensemble fortement résistant (bât. 2, 50 x 40 m) apparaît, non pas en bordure d'un axe principal mais plutôt le long d'un tracé secondaire (axe G) qui le contourne légèrement au sud. Enfin, au carrefour des axes principaux $\mathrm{A}$ et $\mathrm{E}$, le troisième grand ensemble (bât. 3) se manifeste par une anomalie quadrangulaire à laquelle est associée une série des petites formes juxtaposées. D'une longueur maximale de 45 mètres pour une largeur d'environ 30 mètres, cette construction composite s'aligne approximativement sur les deux axes qui l'encadrent $\left(38^{\circ}\right.$ et $128^{\circ}$ pour le bâtiment et $32^{\circ}$ et $133^{\circ}$ pour les axes $\mathrm{A}$ et $\mathrm{E}$ ).

Si la zone centrale, qui se développe d'est en ouest en formant une série de modules réguliers, semble difficile à interpréter au regard des connaissances actuelles, il est néanmoins nécessaire de la considérer comme une zone potentielle de constructions de qualité, voire comme le centre plus urbanisé de l'agglomération.

À l'extrémité est de ce secteur, en limite de la zone prospectée, des anomalies curvilignes et parallèles entre elles sont visibles mais restent peu aisées à interpréter (bât. 4). Cette forme semi-circulaire, visible sur la carte à $0-50 \mathrm{~cm}$ mais quasiment absente aux deux autres profondeurs, méritera d'être complétée par la poursuite de la prospection électrique, même si un cliché aérien documente déjà cette anomalie.

Le reste des anomalies résistantes, visibles sur l'ensemble des trois cartes de résistivité, documente de petites constructions, soit isolées au sein d'îlots (bât. 5) (accessibles par des chemins non visibles?), soit en position de carrefour et 
Figure 6: (Voir planche couleur) Restitution des éléments de construction (grands et petits bâtiments, constructions indéterminées) à partir des prospections électrique et aérienne.

Figure 6: (See colour plate) Restitution of building elements (large and small buildings, indeterminate constructions) from resistivity survey and aerial photography.

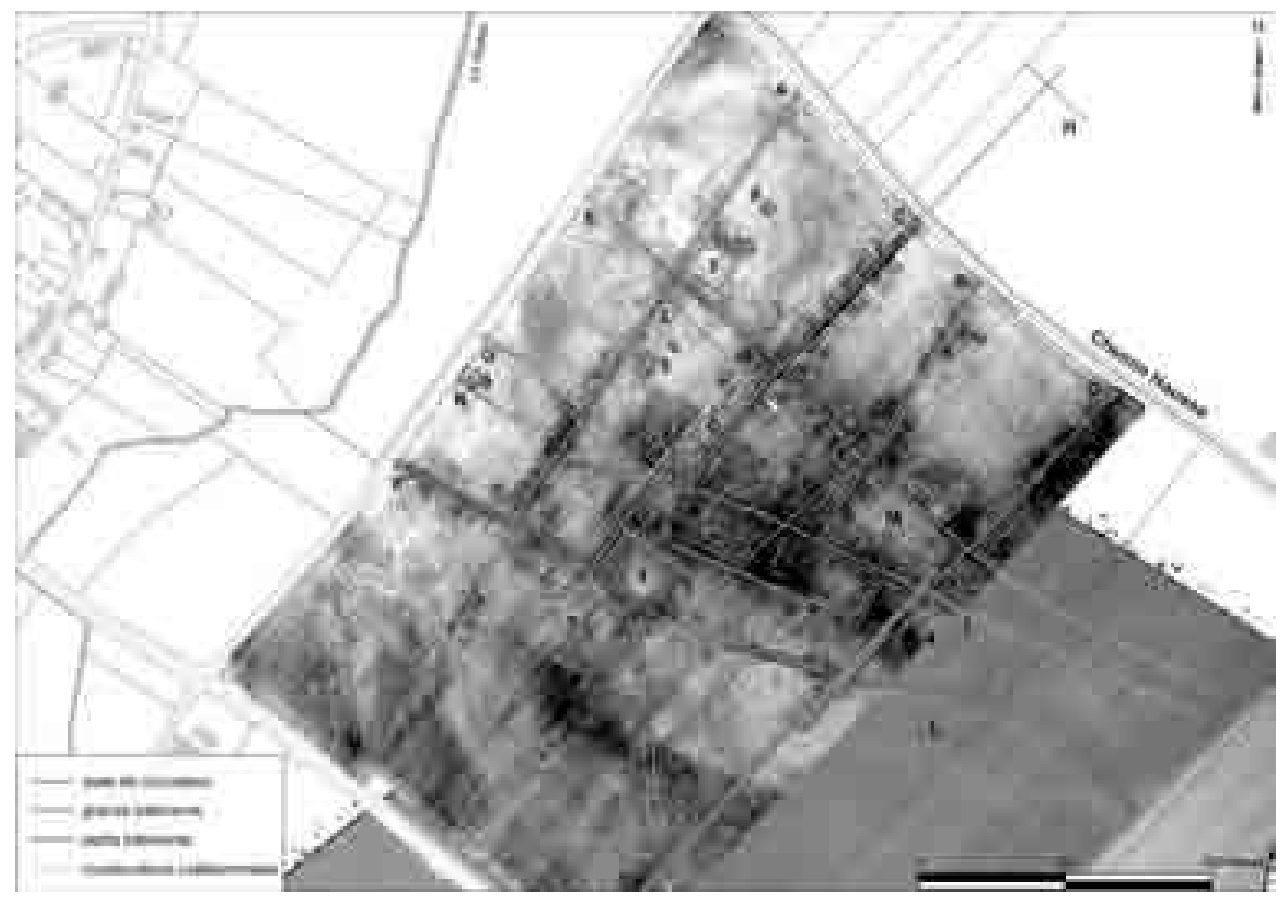

orientées par rapport à un axe principal (bât. 6) ou bien encore au débouché de certains axes, en limite de l'espace urbanisé (bât. 7). Cette catégorie de petits bâtiments est également représentée par une série d'anomalies diffuses, principalement alignées sur les axes $\mathrm{B}, \mathrm{C}, \mathrm{D}$ et $\mathrm{F}$. De faible extension, elles occupent une bande d'une vingtaine de mètres au maximum de chaque côté d'un axe de circulation, laissant l'intérieur des îlots apparemment vide de toute anomalie résistante et donc de constructions maçonnées.

Enfin, quelques anomalies moins résistantes apparaissent en périphérie des espaces fortement résistants. Deux petites constructions carrées de 8 mètres de côté pour la plus septentrionale (bât. 8) et 12 mètres pour la plus occidentale (bât. 9), peuvent être identifiées.

Ainsi, la répartition générale des anomalies permet de distinguer trois types d'organisation de l'espace urbain :

- un centre densément bâti (constructions maçonnées de grande dimension) inscrit à l'intérieur d'un quadrilatère formé par les axes $\mathrm{A}, \mathrm{E}$ et $\mathrm{F}$ et dont la limite est reste encore à d écouvrir;

- une occupation de bord de voies illustrée par des séries de modestes constructions orientées et alignées en fonction d'un axe de circulation (B, C, D et F);

- une occupation plus diffuse en périphérie, à l'ouest de l'axe $A$ et au sud de l'axe $F$.

\section{Organisation interne des constructions}

L'organisation interne des constructions apparaît dépendante de la qualité des bâtiments et de leur étendue. La catégorie des grandes constructions et ensembles de constructions peut être étudiée plus en détail, notamment, les trois ensembles principaux :

- Le complexe quadrangulaire sud (bât. 1) : deux murs parallèles visibles sur ses côtés nord, sud et probablement est (galerie à portique?) entourent un espace intérieur ouvert, vide de toute construction maçonnée (cour, esplanade?). Dans le tiers ouest de cet espace, un ensemble de trois édifices de plan carré d'environ 4,50 $\mathrm{m}$ de côté (temples?) est ceint par un mur de clôture $(25 \times 10 \mathrm{~m})$ délimitant un espace réservé.

- Le complexe central (bât. 2) : plus difficile à décrire, il semble composé d'une série de bâtiments ou pièces dont la façade principale pourrait être celle donnant sur l'axe secondaire G.

- Le complexe nord (bât. 3) : d'aspect bipartite, il apparaît divisé en un grand espace ouvert au sud entouré par un mur de clôture sur ses trois côtés et une série de bâtiments ou pièces de même orientation au nord.

- L'édifice semi-circulaire (bât. 4) peut être délimité par la présence de deux murs courbes d'une trentaine de mètres de long, longueur qui pourrait être doublée si l'on admet l'hypothèse d'un diamètre total restitué d'environ 45 mètres (dans l'éventualité de la présence d'un édifice de spectacle). 
- L'organisation interne des petites constructions reste très difficile à déterminer, à l'exception des deux bâtiments carrés (8 et 9) qui s’apparentent à des édifices à plan centré (temples type "fanum»?).

\section{Organisation générale DE L'AGGLOMÉRATION}

Les trois méthodes de prospection mises en œuvre sur le site apportent des informations complémentaires. La cartographie et le croisement, au sein du SIG, des différents résultats obtenus constituent les bases de l'étude du plan d'urbanisme permettant la reconnaissance quasi complète de la ville (limites, organisation interne, fonction des espaces, réseau de rues...).

L'ensemble des vestiges s'insère dans une trame viaire qui, à première vue, suggérait une organisation souple du plan urbain, mais qui, après analyse de son orientation, dévoile une certaine régularité. Trois orientations préférentielles dominent ce réseau (fig. 5) :

- les deux premières (axes A, B, C et D) matérialisées par deux couples nord-ouest/sud-est présentent deux orientations légèrement différentes $\left(32^{\circ}\right.$ et $\left.43^{\circ}\right)$ qui suggèrent deux planifications vraisemblablement différées dans le temps.

- la troisième orientation (nord-ouest/sud-est) est surtout illustrée par l'axe $\mathrm{E}$ (orienté à $133^{\circ}$ ) et sa parallèle (H) qui peut être restituée au nord à partir d'un court tronçon (environ 60 mètres de long) visible sur un cliché aérien et présentant la même orientation.

La complémentarité des données issues de la prospection aérienne permet de restituer, avec toutefois une certaine incertitude liée à l'imprécision du positionnement des photographies dans le SIG, le prolongement de plusieurs axes au-delà des données fournies par la prospection électrique (ex. des axes C, D, E, F et G) (fig. 7).

Le passage du Chemin Haussé, voie interrégionale, au nord de l'agglomération a certainement dû conditionner l'implantation de la trame urbaine mais n'en a pas été le point central de développement puisqu'aucune anomalie n'a été détectée le long de son tracé. Vraisemblablement en raison d'une topographie plus favorable au sud de cet axe, l'occupation antique a profité d'un vaste espace relativement plan et en bordure à la fois d'un axe de communication important et d'un cours d'eau, le Radon, affluent de la Dives vers lequel trois à quatre axes $(\mathrm{E}, \mathrm{F}, \mathrm{G}$ et $\mathrm{K})$ se dirigent.

Les îlots, a priori non réguliers, composant cette agglomération ne semblent pas densément occupés à l'exception du secteur central. De même, il est envisageable que de nombreuses constructions, notamment privées, aient été réalisées en matériaux périssables, et soient donc peu visibles ou absentes sur l'image de résistivité électrique. Les grandes constructions, bâties en pierres, peuvent sans trop de difficulté être attribuées au domaine public. La comparaison entre ces zones fortement résistantes et la répartition spatiale du mobilier de prospection pédestre conforte l'argument d'une architecture maçonnée dans la mesure où la majorité des artefacts lapidaires est localisée à l'emplacement des grandes constructions et du secteur central (fig. 7). Une densité importante de scories de fer se retrouve surtout dans le quart sud-ouest de la parcelle, en périphérie de l'agglomération, ainsi qu'en association avec les nombreuses constructions reparties le long des axes de circulation $B, C, D$ et $F$ (fig. 3). L'utilisation de ces bâtiments à des fins artisanales est à envisager, au même titre que l'espace non bâti à l'arrière de ces ensembles.

Si le complexe quadrangulaire sud (bât. 1) peut être identifié comme un probable lieu de culte et/ou de rassemblement vers lequel convergent plusieurs axes de circulation, l'édifice semi-circulaire en limite orientale de la prospection électrique nécessite une interprétation plus réservée. En effet, bien que les éléments visibles sur certains clichés aériens suggèrent la présence d'un édifice de spectacle, seule la fouille permettra de vérifier cette hypothèse. Ces deux édifices, caractéristiques de la parure urbaine monumentale antique, demandent aujourd'hui d'approfondir la recherche de modèles semblables en Gaule. La ressemblance du complexe quadrangulaire de Fontaine-les-Bassets avec le sanctuaire monumental de l'antique Gisacum (Vieil-Evreux, Eure : Guyard et Lepert, 1999; Dabas et al., 2005) ou bien celui de Guichainville (Eure : Flotté, 1997) étayerait l'hypothèse d'un édifice cultuel. Bien que sa forme se rapproche beaucoup plus de celle du sanctuaire de Guichainville (galerie périphérique, ensemble de trois édifices carrés à l'intérieur d'un mur d'enceinte), le complexe cultuel de Fontaineles-Bassets reprend, dans de plus modestes dimensions, la même répartition tripartite que celui du Vieil-Evreux. De plus, l'association d'un théâtre et de thermes à un édifice dédié au(x) culte(s) ne doit pas être exclue pour Fontaineles-Bassets (bât. 3 : thermes?, bât. 4 : théâtre?). L'exemple du site de Berthouville (Eure : Lepert, 2008), bien qu'en contexte rural isolé, où un sanctuaire comprenant un péribole d'environ $4800 \mathrm{~m}^{2}(80 \times 60 \mathrm{~m})$ est associé à un théâtre (70 x $50 \mathrm{~m}$ ), se rapproche également des hypothèses avancées pour Fontaine-les-Bassets. Fouillé au XIX ${ }^{\mathrm{e}}$ siècle, le site de Berthouville a récemment fait l'objet d'une prospection électrique dont les résultats ont révélé des anomalies semblables (sanctuaire à deux temples avec galeries à portique, édifice de spectacle) à celles de Fontaine-les-Bassets. L'édifice de spectacle supposé à Fontaine-les-Bassets, de dimensions 
Figure 7 : (Voir planche couleur) Complémentarité des méthodes de prospection aérienne, pédestre (localisation des éléments lapidaires) et électrique.

Figure 7: (See colour plate) Complementary approach of the aerial photography, field survey (location of stone artifacts) and resistivity survey.

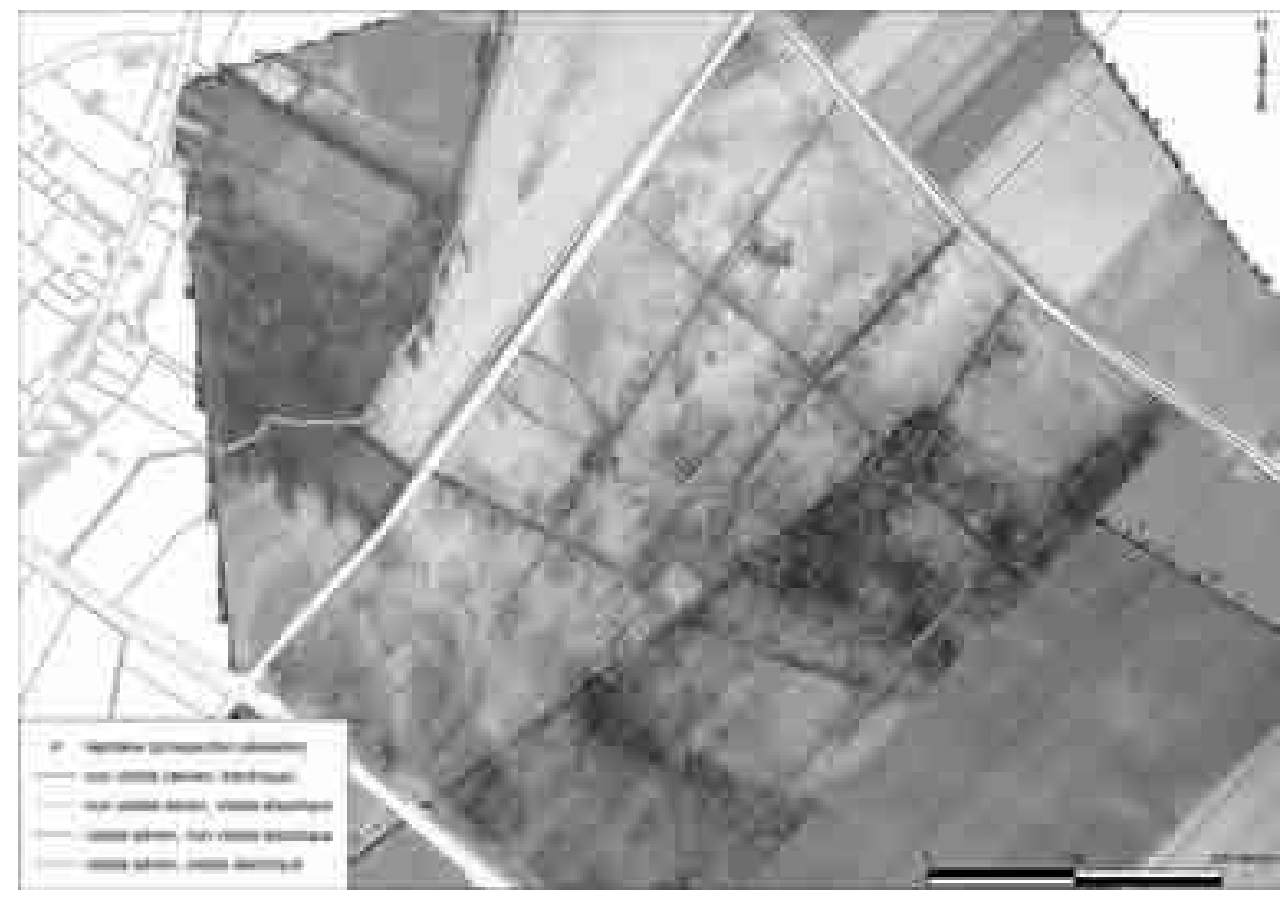

plus modestes (environ 45 mètres de diamètre) serait à classer parmi les édifices de spectacle ou théâtre ruraux galloromains semblables à celui d'Argentomagus (St-Marcel, Indre : Dumasy, 2000), Valognes (Manche : Matter, 1992), St-Désir (Calvados : Lemaître, 1985) ou de HauteNormandie (Matter, 1992). Des éléments d'interprétation supplémentaires devraient être apportés par la poursuite, en 2012, de la prospection électrique vers l'est de l'agglomération de Fontaine-les-Bassets. Ces éléments complèteront les résultats obtenus sur près de $200 \mathrm{~m}^{2}$ lors des premiers sondages réalisés en 2011 (fig. 8).

\section{Conclusion}

Ainsi, les résultats de la prospection électrique ouvrent aujourd'hui, de par leur précision et leur niveau de détail, de nouvelles perspectives quand à l'étude de l'urbanisme de cette agglomération antique. Toutefois, la confrontation des trois méthodes d'investigation utilisées soulève autant de nouveaux questionnements qu'elle n'apporte de réponse. La prospection électrique a permis d'étendre la complexité de ce site au regard de son statut de petite agglomération rurale. C'est désormais toute la réflexion sur la place de cette ville et son rôle au sein d'un territoire encore peu connu pour la période antique qui émerge de ces nouvelles données. La superficie du site, estimée à une vingtaine d'hectares, ainsi que la présence d'édifices publics monumentaux sont autant d'éléments en faveur d'une réévaluation de son rôle au sein du réseau des agglomérations antiques régionales.

Ce sont autant d'interrogations qu'il est possible de formuler à partir des nouvelles données acquises auxquelles seule la fouille pourra permettre de répondre en apportant notamment des éléments de datation (préexistence d'une occupation antérieure, question de l'implantation d'un ou plusieurs réseaux viaires, évolution de la ville...). Si la méthode géophysique est un des moyens les plus rapides et les plus aisés d'obtenir de nouvelles données sur les plans d'urbanisme (les informations nécessitant toutefois un important travail d'interprétation), l'image géophysique ne se substitue en aucun cas au plan de fouille. De même, cette image ne permet d'appréhender que les derniers états de construction et les plans obtenus peuvent parfois être le reflet d'états non contemporains.

L'exploitation des résultats issus des prospections n'est toutefois pas limitée à la simple cartographie des anomalies phytographiques ou électriques et pourra, à l'avenir, permettre une analyse spatiale approfondie à l'issue de la dernière campagne de prospection électrique nécessaire à l'obtention d'un corpus complet sur l'ensemble de l'agglomération. La restitution des éléments observés, certes géolocalisée mais néanmoins approximative quand à la forme et au positionnement exact des vestiges, n'atteint pas encore la précision des relevés issus de la fouille mais peut néanmoins guider les choix à venir quand aux stratégies de fouille à mettre en œuvre. De la précision et de la pertinence des méthodes de 
prospection a dépendu la localisation des sondages réalisés en 2011 (Fig.8), démarche nécessaire à la vérification de l'état de conservation des vestiges mais également à la compréhension historique du site.

Lidée, peut-être encore prématurée, de l'existence d'un édifice de spectacle en limite est de l'image issue de la prospection électrique changerait, si elle se vérifiait, considérablement la vision de l'agglomération antique. En effet, aucun théâtre antique n'a encore été découvert dans le département de l'Orne pour la cité des Esuviens/Sagiens à laquelle appartient le site.

L'absence d'anomalies électriques dans certains secteurs, attestant la présence de constructions non maçonnées, associée aux résultats de la prospection pédestre (abondance des scories de fer) indiquerait la présence de quartiers à vocation artisanale et/ou commerciale. Les structures chauffées ou fossoyées, notamment les fours et aires de combustion, caractéristiques de ce type d'activités pourraient faire l'objet d'une nouvelle approche. Le recours à la prospection magnétique est un moyen envisagé pour affiner la connaissance de ces espaces et ainsi compléter la vision de cette agglomération dont il est évident qu'elle n'était pas uniquement composée de somptueux édifices en pierres. La complémentarité de ces deux approches (électrique et magnétique), bien souvent mise en ouvre sur d'autres sites antiques (Neubauer, 2001), serait dans l'exemple de Fontaine-les-Bassets une voie à explorer quant à l'étude de l'architecture vernaculaire, souvent méconnue en milieu urbain.

\section{Remerciements}

L'auteur remercie tout particulièrement Michel Dabas ainsi que Thomas Jubeau (Geocarta) pour leur aide précieuse et avertie quant à l'analyse des données de la prospection électrique et sa traduction dans la langue des archéologues. Merci également à Jean Desloges pour les nombreuses photographies aériennes dont il est l'auteur ainsi qu'à Gilles Guillemot et Guy Leclerc pour avoir partagé leur connaissance du site.

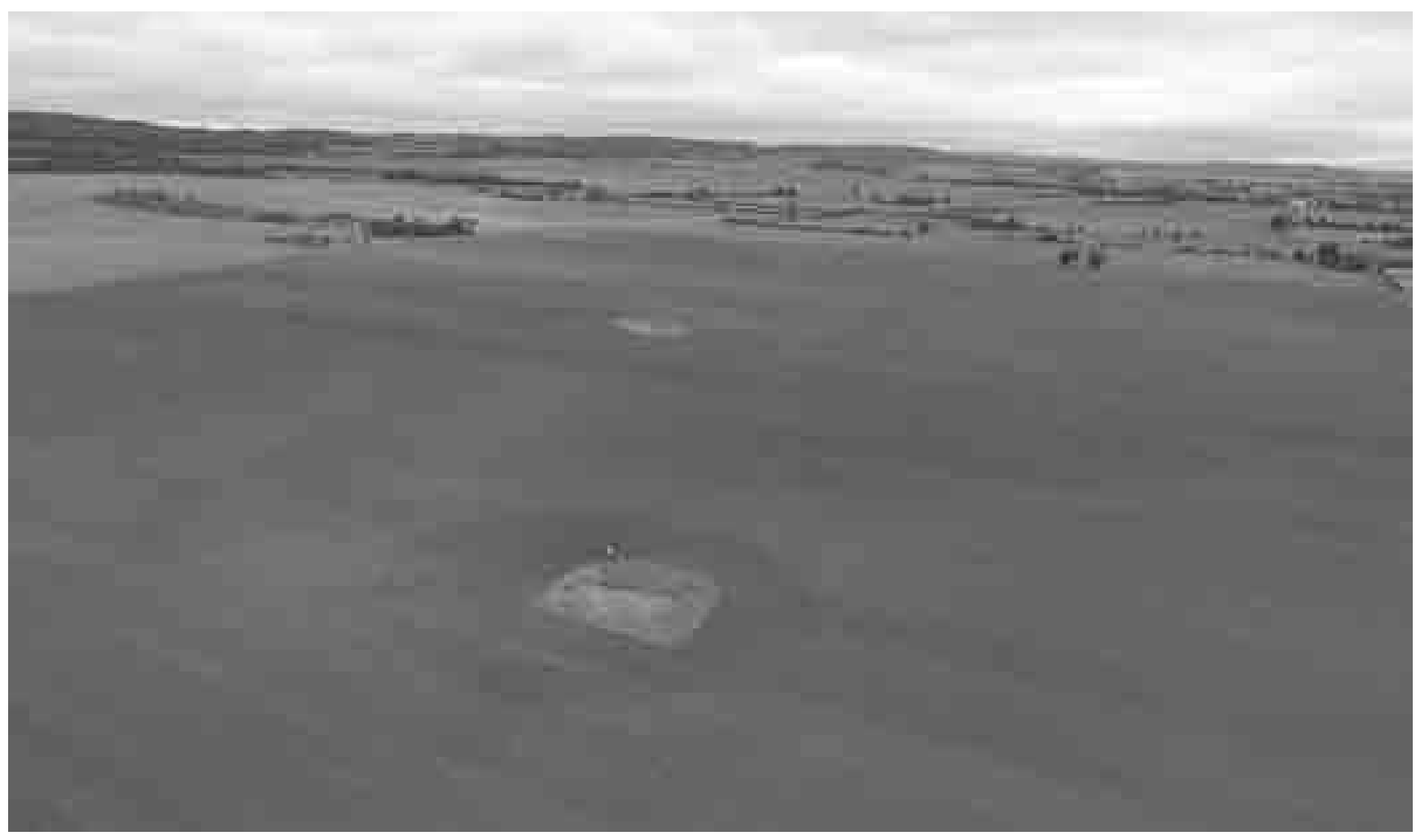

Figure 8 : Vue aérienne de l'agglomération antique (cliché pris du sud-ouest par cerf-volant, auteur : F. Levalet) et implantation des sondages 2011.

Figure 8: Aerial picture of the Roman town (taken by kite from the southwest, author: F. Levalet) and location of the 2011 excavation. 


\section{Bibliographie}

CAmpana, S., 2006. DGPS e mobile GIS per l'archeologia dei paesaggi, dans Laser scanner e GPS : paesaggi archeologici e tecnologie digitali 1, atti del workshop, 3 marzo 2005 Grosseto, All'Insegna del Giglio, Firenze, p. 201-225.

Dabas, M., Guyard, L. et Lepert, Th., 2005. Gisacum revisité. Dossiers archéologie et sciences des origines, 308, p. 52-61.

Dabas, M., 2006. La prospection géophysique. In : Ferdière (dir.). La prospection. Errance, Paris, p. 167-216.

Desloges, J., 1994. Prospection aérienne dans les plaines de grande culture du Calvados et de l'Orne, Bilan scientifique régional, Basse-Normandie (1993), p. 102.

Desloges, J., 2001. Chemins et paysages de la Plaine de Caen. Prospection aérienne, Bilan scientifique régional, BasseNormandie (2000), p. 99-101.

Desloges, J., 2011. La prospection aérienne de la Plaine de Caen. Bilan de la recherche archéologique en Basse-Normandie, 1984-2004, L'antiquité,p . 130-141.

Desloges, J., Maneuvrier, Ch. et Forfait, N.,1993. L'apport de la prospection aérienne à l'étude du peuplement antique. La Dives, frontière antique? Bulletin du groupe pour la recherche archéologique et la prospection en Pays d'Auge,p . 24-28.

Dieudonné-Glad, N., 2010. Vendeuvre-du-Poitou, Les Tours Mirandes. Bilan scientifique régional, Poitou-Charentes (2009), p. 167-168.

Dumasy, F., 2000. Le théâtre d'Argentomagus (Saint-Marcel, Indre), Paris, Éd. de la Maison des sciences de l'homme.
Flotté, P., 1997. Guichainville, « Le devant de la Garenne », Bilan scientifique régional, Haute-Normandie (1996),p . 37-39.

Guyard, L. et Lepert, Th., 1999. Renaissance d'une ville sanctuaire gallo-romaine : le Vieil-Évreux, Archéologia, 359, p. 24.

LeClerC, G., 1996. Les sites ruraux gallo-romains de l'Orne. Rapport de prospection archéologique, déposé au Service régional de l'archéologie de Basse-Normandie, p. 126-136.

Lemaître, C., 1985. A propos du théâtre gallo-romain du VieuxLisieux, Le Pays d'Auge, 4, p. 9-16.

Lepert, Th., 2008. Berthouville, Villeret, Bilan scientifique régional, Haute-Normandie (2005),p . 18-19.

Matter, M., 1992. Particularités architecturales des édifices de spectacles en Gaule Lyonnaise, dans Landes, CH., et Kramérovskis, V. Spectacula 2. Le théâtre antique et ses spectacles. Actes du colloque tenu au Musée archéologique Henri Prades de Lattes les 27, 28, 29 et 30 avril 1989, Lattes, Musée archéologique Henri Prades, p. 29-36.

Neubauer, W., 2001. Images of the invisible-prospection methods for the documentation of threatened archaeological sites, Naturwissenschaften, 88, p. 13-24.

Rodier, X., et Saligny, L., 2006. Utilisation de GPS en prospection, dans Ferdière (dir.), La prospection, Paris, Errance, p. 13-19.

Thivet, M., Bossuet, G. et Laplaige, C., 2009. Integrated geophysical and LIDAR surveys at the archaeological site of Ancient Epomanduodurum, Mandeure-Mathay (FrancheComté, Eastern France). ArchéoSciences, 33, p. 151-154. 